\title{
Inhalational versus intravenous magnesium sulfate in management of pulmonary hypertension in neonates
}

\author{
Amany Ahmad Abdalsalam, Ramadan A. Mahmoud, \\ Mohamed Abd.ELAal Mohamed, Soumaya El-sayed Hadhoud \\ Departments of Pediatrics, Faculty of Medicine, Sohag University
}

\begin{abstract}
Background: persistent pulmonary hypertension of the newborn (PPHN) is a serious medical emergency in the neonatal period which occurs because of the failure of the transition of the fetal circulation into the normal circulation. The condition is characterized by persistently elevated pulmonary vascular pressures and despite numerous modalities of treatment available, the condition carries with it a high rate of mortality and morbidity.
\end{abstract}

Objective: To evaluate the response to inhaled versus intravenous magnesium sulfate (MgSO4) in the treatment of pulmonary hypertension in neonates.

Methodology: This is an observational prospective study done in Sohag university hospital in the neonatal intensive care unit during the period from January 2016 to December 2017 on 50 neonates who were diagnosed to have PPHN by echocardiography. In this study, we compared the effect of intravenous versus the inhalational MgSO4 on reducing the PHN and SILDENAFIL was used as an adjuvant treatment.

Results: there was a statistically significant difference between the three studied groups as regard: age ( $\mathrm{P}$ value 0.034$)$, sex (P-value 0.001$)$, the degree of respiratory distress (P-value 0.01), the pulmonary artery pressure before treatment (P-value $<0.001$ ), side effects of treatment (P-value 0.012), duration of treatment (P-value $<0.001$ ) and the $\mathrm{SpO} 2$ after treatment (P-value 0.005). Also there was a great difference in $\mathrm{SpO} 2$ in the right hand before and after treatment; $\mathrm{P}$ value was $<0.001$ in group A and 0.002 in both group B and C. Pulmonary artery pressure as well differs greatly before and after treatment in the three groups; $\mathrm{P}$ value was $<0.001$ in group A and 0.002 in both group B and C.

Conclusion: Based on this study we don't recommend the use of nebulized MgSO4 alone as a treatment of PPHN in neonates (as no improvement occurred in patients used it alone).

Keywords: Inhalational, Intravenous magnesium sulfate, management of pulmonary hypertension in neonates.

\section{Introduction}

Pulmonary hypertension of the newborn occurs in 1.9 per 1000 live births and affected infants are hypoxaemic because of right-to-left shunts through the ductus arteriosus and foramen ovale. Pulmonary hypertension of the new-born may be primary, or secondary to a variety of conditions [1].
The primary form of the disease presents soon after birth with hypoxemia in a baby with normal lungs clinically and radiologically. Usually, it is associated with high-risk pregnancies as diabetes mellitus, hypertension, and maternal indomethacin intake. Secondary causes include congenital heart malformations 
(most commonly associated with obstruction to pulmonary venous drainage), high output cardiac failure from large arteriovenous malformations and persistent pulmonary hypertension of the newborn (PPHN); of these, the most common cause is PPHN [2].

PPHN develops when pulmonary vascular resistance (PVR) remains elevated after birth, resulting in the right-to-left shunting of blood through fetal circulatory pathways. The PVR may remain elevated due to pulmonary hypoplasia, like that seen with a congenital diaphragmatic hernia; maldevelopment of the pulmonary arteries, seen in meconium aspiration syndrome; and maladaptation of the pulmonary vascular bed as occurs with perinatal asphyxia. Infants with PPHN have cyanosis, tachypnea, acidaemia, normal pulmonary parenchymal markings on the chest radiograph, and anatomically normal hearts [3].

Doctors have been using magnesium sulfate (MgSO4) for many years in the treatment of toxemia of pregnancy. It is a natural calcium antagonist. It acts on the metabolism of prostaglandin, suppresses the release of catecholamines, reduces the responsiveness of smooth muscles to vasopressors. Also, MgSO4 at high concentration is a potent vasodilator and can prevent and reduce pulmonary hypertension caused by hypoxia, sedative, muscle relaxant and has an antithrombotic action on the brain and kidney $[\mathbf{4}, \mathbf{5}]$.

$\mathrm{MgSO} 4$ is a safe and cheaper alternative for first-line treatment in moderate PHN and was chosen to be an alternative therapy for PHN when other conventional treatments fail, contraindicated or not available. Magnesium sulfate can dilate the constricted muscles in the pulmonary arteries. However, its action is not specific and when given via an intravenous infusion, it will act on other muscles in the body including other arteries. This means that even if it was found to be effective in pulmonary hypertension, unwanted actions in other parts of the body might be a problem [4]. However, inhaled magnesium sulfate has not been studied yet for the treatment of pulmonary hypertension.

Sildenafil is a phosphodiesterase type 5 inhibitor that reduces pulmonary vascular resistance selectively. It also is great in the treatment of infants with PPHN. It is available now in both oral and intravenous forms; however, there is an increased risk of systemic vasodilation with the I.V rout.

\section{Aim of the work}

To evaluate the response of inhaled versus intravenous magnesium sulfate (MgSO4) in the treatment of pulmonary hypertension in neonates.

\section{Patients and Methods}

Place of the study: Neonatal intensive care unit (N.I.C.U) at Sohag University Hospital.

Type of the Study: Prospective hospital-based study.

Study Period: From January 2016 to

December 2017.

\section{Patients:}

\section{Inclusion Criteria:}

50 full-term or near term neonates who were diagnosed to have pulmonary hypertension (estimated pulmonary artery pressure $<50$ $\mathrm{mmHg}$ ) presented to N.I.C.U during the period of the study were included in the study.

\section{Exclusion Criteria:}

- Preterm babies > 34 weeks.

- Pulmonary hypertension associated with lung malformations.

- Pulmonary hypertension associated with diaphragmatic or hiatus hernia.

- Pulmonary hypertension patients on mechanical ventilation.

\section{Ethical consideration:}


Approval of the Sohag Faculty of Medicine Research Ethics Committee was obtained. Written consent was obtained from guardians.

\section{Methods of the study:}

Patients included in the study were subjected to the following (diagnostic criteria of pulmonary hypertension):

- Full antenatal and postnatal histories.

- Full clinical examination: including general examination, chest and abdominal examination with emphasize on the cardiac examination including the apex site, 1 st, and 2nd heart sounds and manifestations of heart failure (tachycardia, tachypnea and enlarged liver).

- Pulse oximeter monitoring in the four limbs.

- Blood pressure monitoring in the four limbs.

- Chest X-ray

- Serial arterial blood gases

- Electrocardiogram: The ECG may provide suggestive or supportive evidence of pulmonary hypertension by demonstrating RV hypertrophy and strain, and right atrial dilatation

- Echocardiogram: measures pulmonary artery pressure, low ventricular output and low ejection fraction and fraction shortening.

The protocol of administration of magnesium sulfate:

Neonates fulfilled the inclusion criteria were divided into three groups:

\section{Group A: (26 patients)}

Received intravenous magnesium sulfate only. A loading dose of 200 $\mathrm{mg} / \mathrm{kg}$ given over 20 minutes followed by a continuous infusion of 50-150 $\mathrm{mg} / \mathrm{kg} / \mathrm{hour}$ and daily Echo was done until reaching pulmonary artery pressure of $30 \mathrm{mmHg}$.

Group B: (12 patients) received inhalational magnesium sulfate $\left(100 \_300 \mathrm{mg}+4 \mathrm{ml}\right.$ sterile water) according to the severity of respiratory distress for 3 times daily with daily Echo follow up but there was no improvement in the general condition or pulmonary pressure, so oral sildenafil was added after 24 hours in a dose of $1 \mathrm{mglkg}$ lday in four divided doses (via the nasogastric tube) with daily Echo follow up until pulmonary artery pressure reached 30 $\mathrm{mmHg}$. And as a result, we decided to change our protocol of administration and added oral sildenafil from the start to patients who receive inhalational $\mathrm{MgSO} 4$ to save the lives of our neonates.

Group C: (12 patients)

Received inhalational MgSO4 (100$300 \mathrm{mg}+4 \mathrm{ml}$ sterile water) according to the severity of respiratory distress for 3 times daily together with oral sildenafil in a dose of $1 \mathrm{mg} \mathrm{kg} / \mathrm{day}$ in four divided doses (via the nasogastric tube) with daily Echo follow up until pulmonary artery pressure reached 30 $\mathrm{mmHg}$.

\section{Statistical analysis:}

Data were analyzed using IBM SPSS Statistics for Windows version 23.0.

Quantitative data were expressed as mean \pm standard deviation, median, and range. Qualitative data were expressed as number and percentage. The data were tested for normality using the Shapiro-Wilk test. The nonparametric Wilcoxon Signed Ranks test and Kruskal-Wallis test were used for data that wasn't normally distributed. One-Way ANOVA test was used for normally distributed data. Chi-square $(\chi 2)$ test and Fisher's Exact Test were used for comparison regarding qualitative variables as appropriate. A 5\% level was chosen as a level of significance in all statistical tests used in the study. 
SOHAG MEDICAL JOURNAL Inhalational versus intravenous magnesium sulfate in management

Vol. 24 No. 1 Jan 2020

Amany Ahmad Abdalsalam

\section{Results:}

\begin{tabular}{|c|c|c|c|c|}
\hline \multirow[t]{2}{*}{ Parameter } & \multicolumn{3}{|l|}{ Group } & \multirow[t]{2}{*}{ P-value } \\
\hline & $\begin{array}{l}\text { I.V MgSo4 } \\
(\mathrm{N}=26) \mathrm{A}\end{array}$ & $\begin{array}{l}\text { Inhalational } \\
\text { MgSo4 then } \\
\text { Sildenafil } \\
(\mathrm{N}=12) \mathrm{B}\end{array}$ & $\begin{array}{l}\text { Inhalational } \\
\text { MgSo4 with } \\
\text { Sildenafil } \\
(\mathrm{N}=12) \mathrm{C}\end{array}$ & \\
\hline $\begin{array}{l}\text { Age (days) } \\
\text { Mean } \pm \text { S.D. } \\
\text { Median } \\
\text { (Range) }\end{array}$ & $\begin{array}{l}2.5 \pm 1.45 \\
2(1-5)\end{array}$ & $\begin{array}{l}4.33 \pm 2.67 \\
4(1-8)\end{array}$ & $\begin{array}{l}1.83 \pm 0.72 \\
2(1-3)\end{array}$ & $0.034 *$ \\
\hline $\begin{array}{l}\text { Sex } \\
\text { Female } \\
\text { Male }\end{array}$ & $\begin{array}{l}13(50 \%) \\
13(50 \%)\end{array}$ & $\begin{array}{l}10(83.3 \%) \\
2(16.7 \%)\end{array}$ & $\begin{array}{l}1(8.3 \%) \\
11(91.7 \%)\end{array}$ & 0.001 \\
\hline
\end{tabular}

Table (1): Comparison between the three studied groups regarding age and sex.

P-value was calculated by Chi-square test

* P-value was calculated by Kruskal Wallis Test

$P$-value $<0.05$ is statistically significant

The mean age in each studied group was as follows: in group A: 2.5 days, in group B 4.33 days and group C 1.83 days. Sex distribution was $50 \%$ male and $50 \%$ female in group A, $16.7 \%$ male, and $83.3 \%$ female in group B and $91.7 \%$ male and $8.3 \%$ female in group $\mathrm{C}$ with statistically significant $\mathrm{P}$ value between the studied groups as regard age and sex.

\begin{tabular}{|c|c|c|c|c|}
\hline \multirow[t]{2}{*}{ Parameter } & \multicolumn{3}{|c|}{ Group } & \multirow[t]{2}{*}{$P$-value } \\
\hline & $\begin{array}{c}\text { I.V MgSO } \\
(\mathrm{N}=26) \\
\mathrm{A} \\
\end{array}$ & $\begin{array}{c}\text { Inhalational } \mathrm{MgSO}_{4} \\
\text { then Sildenafil } \\
(\mathrm{N}=12) \\
\text { B }\end{array}$ & $\begin{array}{c}\text { Inhalational } \mathrm{MgSO}_{4} \\
\text { with Sildenafil } \\
(\mathrm{N}=12) \\
\mathrm{C} \\
\end{array}$ & \\
\hline $\begin{array}{l}\text { Degree of RD } \\
\text { First } \\
\text { Second } \\
\text { Third } \\
\text { Fourth } \\
\end{array}$ & $\begin{array}{c}0(0.0 \%) \\
7(26.9 \%) \\
9(34.6 \%) \\
10(38.5 \%) \\
\end{array}$ & $\begin{array}{c}1(8.3 \%) \\
8(66.7 \%) \\
3(25 \%) \\
0(0.0 \%) \\
\end{array}$ & $\begin{array}{l}0(0.0 \%) \\
2(16.7 \%) \\
8(66.6 \%) \\
2(16.7 \%) \\
\end{array}$ & 0.01 \\
\hline $\begin{array}{l}\text { Congenital } \\
\text { anomalies } \\
\text { Down syndrome } \\
\text { Hydrocephalus } \\
\text { Multiple } \\
\text { No } \\
\end{array}$ & $\begin{array}{c}0(0.0 \%) \\
1(3.8 \%) \\
1(3.8 \%) \\
24(92.4 \%)\end{array}$ & $\begin{array}{c}\mathbf{0}(\mathbf{0 . 0 \%}) \\
\mathbf{0}(0.0 \%) \\
0(0.0 \%) \\
12(100 \%)\end{array}$ & $\begin{array}{c}1(8.3 \%) \\
0(0.0 \%) \\
0(0.0 \%) \\
11(91.7 \%)\end{array}$ & 0.534 \\
\hline $\begin{array}{l}\mathrm{SpO}_{2}(\%)(\mathrm{RT} \\
\text { hand) } \\
\text { Mean } \pm \text { S.D. } \\
\text { Median (Range) } \\
\end{array}$ & $\begin{array}{c}79.15 \pm 8.18 \\
80.5(60-90)\end{array}$ & $\begin{array}{l}83.42 \pm 3.15 \\
83(79-90)\end{array}$ & $\begin{array}{c}79.92 \pm 5.19 \\
80.5(70-86)\end{array}$ & $0.251^{*}$ \\
\hline $\begin{array}{l}\mathrm{SpO}_{2}(\%)(\mathrm{LT} \\
\text { hand) } \\
\text { Mean } \pm \text { S.D. } \\
\text { Median (Range) } \\
\end{array}$ & $\begin{array}{c}68.58 \pm 7.91 \\
69.5(50-78)\end{array}$ & $\begin{array}{l}72.75 \pm 2.77 \\
72(69-78)\end{array}$ & $\begin{array}{l}69.33 \pm 5.21 \\
70(60-76)\end{array}$ & $0.223 *$ \\
\hline $\begin{array}{l}\text { Heart sounds } \\
\text { Normal } \\
\text { Single loud S2 } \\
\end{array}$ & $\begin{array}{c}20(76.9 \%) \\
6(23.1 \%)\end{array}$ & $\begin{array}{c}10(83.3 \%) \\
2(16.7 \%)\end{array}$ & $\begin{array}{c}10(83.3 \%) \\
2(16.7 \%)\end{array}$ & 0.75 \\
\hline $\begin{array}{l}\text { Murmur } \\
\text { Harsh systolic } \\
\text { No }\end{array}$ & $\begin{array}{l}10(38.5 \%) \\
16(61.5 \%)\end{array}$ & $\begin{array}{l}5(41.7 \%) \\
7(58.3 \%)\end{array}$ & $\begin{array}{l}4(33.3 \%) \\
8(66.7 \%)\end{array}$ & 0.913 \\
\hline
\end{tabular}

Table(2): Compari son between the three studied groups regardin g the neonatal examinat ion. (Before treatmen t)

P-value was calculated by Chi-square test * P-value was calculated by Kruskal Wallis Test $\mathrm{P}$-value $<0.05$ is statistically significant 
As regards neonatal examination, respiratory distress was obvious in all cases but differs in its degree; in the group, A $0.0 \%$ of cases had RD1, $26.9 \%$ of cases had RD2, $34.6 \%$ of cases had RD3 and $38.5 \%$ of cases had RD4. In group B $8.3 \%$ of cases had RD1, 66.7\% had RD2, 25\% had RD3 and $0.0 \%$ had RD4. In group C $0.0 \%$ had RD1, 16.7\% had RD2, 66.6\% had RD3 and $16.7 \%$ had RD4, with statistically significant P-value between the three groups.

Congenital anomalies were found in 3 cases only out of 50 , two cases were in group A: one had hydrocephalus and the other had multiple congenital anomalies. And one case was in group C who was Down syndrome.

The mean value of oxygen saturation in group A was $79.15 \%$ in the right hand and $68.58 \%$ in the left hand, while in group B was $83.42 \%$ in the right hand and $72.75 \%$ in the left hand, and in group $\mathrm{C}$ was $79.92 \%$ in the right hand and $69.33 \%$ in the left hand.

Heart sounds were normal in $76.9 \%$ of group A, $83.3 \%$ of group B and also $83.3 \%$ of group $\mathrm{C}$, while single loud S2 was heard in $23.1 \%$ of group A, $16.7 \%$ of group B and also $16.7 \%$ of group C.

Harsh systolic murmur was heard in $38.5 \%$ of group A, $41.7 \%$ of group B and $33.3 \%$ of group C.

$\mathrm{P}$-value was statistically insignificant as regards the presence of congenital anomalies, oxygen saturation in right and left hands, heart sounds and heart murmurs.

\begin{tabular}{|c|c|c|c|c|}
\hline \multirow[t]{2}{*}{ Parameter } & \multicolumn{3}{|c|}{ Group } & \multirow[t]{2}{*}{ P-value } \\
\hline & $\begin{array}{c}\text { I. } \mathrm{V} \mathrm{MgSO}_{4} \\
(\mathrm{~N}=26) \\
\mathrm{A}\end{array}$ & $\begin{array}{c}\text { Inhalational } \\
\text { MgSO }_{4} \\
\text { then } \\
\text { Sildenafil } \\
(\mathrm{N}=12) \\
\text { B } \\
\end{array}$ & $\begin{array}{c}\text { Inhalational } \\
\mathrm{MgSO}_{4} \\
\text { with } \\
\text { Sildenafil } \\
(\mathrm{N}=12) \\
\mathrm{C} \\
\end{array}$ & \\
\hline $\begin{array}{l}\text { Blood glucose } \\
\text { Hypoglycemia } \\
\text { Normal } \\
\end{array}$ & $\begin{array}{c}3(11.5 \%) \\
23(88.5 \%) \\
\end{array}$ & $\begin{array}{c}0(0.0 \%) \\
12(100 \%) \\
\end{array}$ & $\begin{array}{c}1(8.3 \%) \\
11(91.7 \%) \\
\end{array}$ & 0.475 \\
\hline \begin{tabular}{l|l} 
CBC \\
Normal \\
Polycythemia \\
Thrombocytopenia \\
\end{tabular} & $\begin{array}{c}22(84.6 \%) \\
1(3.8 \%) \\
3(11.5 \%) \\
\end{array}$ & $\begin{array}{c}12(100 \%) \\
0(0.0 \%) \\
0(0.0 \%) \\
\end{array}$ & $\begin{array}{c}11(91.7 \%) \\
1(8.3 \%) \\
0(0.0 \%) \\
\end{array}$ & 0.401 \\
\hline $\begin{array}{l}\text { Serum } \\
\text { electrolytes } \\
\text { Hypocalcaemia } \\
\text { Normal } \\
\end{array}$ & $\begin{array}{c}2(7.7 \%) \\
24(92.3 \%)\end{array}$ & $\begin{array}{c}2(16.7 \%) \\
10(83.3 \%)\end{array}$ & $\begin{array}{c}1(8.3 \%) \\
11(91.7 \%)\end{array}$ & 0.676 \\
\hline \begin{tabular}{l|} 
ABG \\
Compensated \\
metabolic acidosis \\
Hypoxemia \\
Uncompensated \\
metabolic acidosis
\end{tabular} & $\begin{array}{c}3(11.5 \%) \\
11(42.3 \%) \\
12(46.2 \%)\end{array}$ & $\begin{array}{c}0(0.0 \%) \\
10(83.3 \%) \\
2(16.7 \%)\end{array}$ & $\begin{array}{l}0(0.0 \%) \\
6(50 \%) \\
6(50 \%)\end{array}$ & 0.109 \\
\hline
\end{tabular}

Table(3): Comparison between the three studied groups regarding laboratory investigations. ( before treatment)

\section{P-value was calculated by Chi-Square Test}

This table shows the results of laboratory investigations before treatment for the cases. As regards blood glucose level; in group A only 3 cases had hypoglycemia ( RBS was 30,
32 and 25 respectively), no cases in group $\mathrm{B}$ and one case in group $\mathrm{C}$.

CBC was normal in the majority of cases apart from one case in both group $\mathrm{A}$ and one case in group $\mathrm{C}$ who 
had polycythemia and three cases in group A had thrombocytopenia.

As regards serum electrolytes; hypocalcemia appears in two cases of group A, two cases of group B and one case of group $\mathrm{C}$, while the rest of the cases had normal serum electrolytes.

The results of the $A B G$ were as follow:

In group A $11.5 \%$ had compensated metabolic acidosis, $46.2 \%$ had uncompensated metabolic acidosis and 42.3\% had hypoxemia only. In group B $83.3 \%$ had hypoxemia and $16.7 \%$ had uncompensated metabolic acidosis. In group C $50 \%$ of cases had hypoxemia and the rest $50 \%$ had uncompensated metabolic acidosis.

$\mathrm{P}$-value was statistically insignificant as regards laboratory investigations in the three studied groups.

\begin{tabular}{|c|c|c|c|c|c|}
\hline \multirow[t]{2}{*}{ Parameter } & \multicolumn{3}{|l|}{ Group } & \multirow[t]{2}{*}{ P-value } & \multirow{5}{*}{$\begin{array}{l}\text { Table(4): } \\
\text { Comparis } \\
\text { on } \\
\text { between } \\
\text { the three } \\
\text { studied } \\
\text { groups } \\
\text { regarding } \\
\text { imaging } \\
\text { findings }\end{array}$} \\
\hline & $\begin{array}{l}\mathrm{I} . \mathrm{V} \mathrm{MgSO} \\
4 \\
(\mathrm{~N}=26) \\
\mathrm{A}\end{array}$ & $\begin{array}{l}\text { Inhalational } \\
\mathrm{MgSO}_{4} \\
\text { then Sildenafil } \\
(\mathrm{N}=12) \\
\text { B }\end{array}$ & $\begin{array}{l}\text { Inhalational } \\
\text { MgSO }_{4} \\
\text { with Sildenafil } \\
(\mathrm{N}=12) \\
\mathrm{C} \\
\end{array}$ & & \\
\hline $\begin{array}{l}\text { Chest X-ray } \\
\text { Bilateral lung } \\
\text { opacities } \\
\text { Cardiomegaly } \\
\text { Hyper expanded } \\
\text { lungs } \\
\text { Lung oligemia } \\
\text { Normal } \\
\text { Wide } \\
\text { mediastinum }\end{array}$ & $\begin{array}{l}1(3.8 \%) \\
2(7.7 \%) \\
1(3.8 \%) \\
6(23.1 \%) \\
15(57.7 \%) \\
1(3.8 \%)\end{array}$ & $\begin{array}{l}0(0.0 \%) \\
1(8.3 \%) \\
0(0.0 \%) \\
0(0.0 \%) \\
11(91.7 \%) \\
0(0.0 \%)\end{array}$ & $\begin{array}{l}0(0.0 \%) \\
0(0.0 \%) \\
0(0.0 \%) \\
5(41.7 \%) \\
6(50 \%) \\
1(8.3 \%)\end{array}$ & $0.392 *$ & \\
\hline \multicolumn{5}{|c|}{ Echocardiography } & \\
\hline $\begin{array}{l}\text { Pulmonary } \\
\text { pressure } \\
\text { Mean } \pm \text { S.D. } \\
\text { Median (Range) }\end{array}$ & $\begin{array}{l}69.81 \pm \\
17.51 \\
70(50- \\
110) \\
\end{array}$ & $\begin{array}{l}57.08 \pm 4.98 \\
60(50-65)\end{array}$ & $\begin{array}{l}78.17 \pm 8.04 \\
80(65-90)\end{array}$ & $<0.001$ & \\
\hline
\end{tabular}

\section{P-value was calculated by Kruskal Wallis Test \\ *P-value was calculated by Chi-square test \\ $P$-value $<0.05$ is statistically significant}

Chest X-ray findings were as follows: in the group, A $3.8 \%$ had bilateral lung opacities, $7.7 \%$ had cardiomegaly, $3.8 \%$ had hyper expanded lungs, $23.1 \%$ had lung oligemia, $3.8 \%$ had wide mediastinum and $57.7 \%$ were normal. In group B only one case had cardiomegaly and the rest of the cases were normal. In group C $41.7 \%$ had lung oligemia, $8.3 \%$ had wide mediastinum and the rest $50 \%$ were normal. P-value was statistically insignificant among the three groups. PAP in echocardiography show statistically significant difference in between the three study groups, in group A the mean was 69.81 while in group B was 57.08 and in the group, $\mathrm{C}$ was 78.17 
SOHAG MEDICAL JOURNAL Inhalational versus intravenous magnesium sulfate in management Vol. 24 No. 1 Jan 2020 Amany Ahmad Abdalsalam

\begin{tabular}{|c|c|c|c|c|c|c|c|}
\hline \multirow{2}{*}{$\begin{array}{l}\text { Side } \\
\text { effects of } \\
\text { treatment }\end{array}$} & \multicolumn{3}{|l|}{ Group } & \multirow{2}{*}{$\begin{array}{l}P \text { - } \\
\text { value }\end{array}$} & \multirow[t]{2}{*}{ P1 } & \multirow[t]{2}{*}{ P2 } & \multirow[t]{2}{*}{ P3 } \\
\hline & $\begin{array}{l}\text { I. } \mathrm{MgSO}_{4} \\
(\mathrm{~N}=26) \\
\mathrm{A}\end{array}$ & $\begin{array}{l}\text { Inhalational } \\
\mathrm{MgSO}_{4} \\
\text { then } \\
\text { Sildenafil } \\
(\mathrm{N}=12) \mathrm{B}\end{array}$ & $\begin{array}{l}\text { Inhalational } \\
\mathrm{MgSO}_{4} \\
\text { with } \\
\text { Sildenafil } \\
(\mathrm{N}=12) \mathrm{C}\end{array}$ & & & & \\
\hline $\begin{array}{l}\text { Hypotensi } \\
\text { on } \\
\text { No }\end{array}$ & $\begin{array}{l}8(30.8 \%) \\
18(69.2 \%)\end{array}$ & $\begin{array}{l}0(0.0 \%) \\
12(100 \%)\end{array}$ & $\begin{array}{l}0(0.0 \%) \\
12(100 \%)\end{array}$ & 0.012 & 0.039 & 0.039 & NA \\
\hline
\end{tabular}

Table(5): Compariso n between the three studied groups regarding the side effects of treatment.

P-value compared the three groups and was calculated by the Chi-Square test. p1 a comparison between group $A$ and group $B$.

$P 2$ a comparison between group $A$ and group $C$.

$P 3$ a comparison between group $B$ and group $C$.

$P$ values were calculated by Fisher's Exact Test

As regards the side effects of treatment, hypotension was the only side effect and occurs in $30.8 \%$ of group $\mathrm{B}$ and group $\mathrm{C}$. P-value shows a group A with no reported cases in both statistically significant difference between the study groups.

\begin{tabular}{|c|c|c|c|c|c|c|c|}
\hline \multirow[t]{2}{*}{ Parameter } & \multicolumn{3}{|c|}{ Group } & \multirow[t]{2}{*}{ P-value } & \multirow[t]{2}{*}{ P1 } & \multirow[t]{2}{*}{$\mathbf{P 2}$} & \multirow[t]{2}{*}{ P3 } \\
\hline & $\begin{array}{c}\text { I.V } \mathrm{MgSO}_{4} \\
(\mathrm{~N}=26) \\
\mathrm{A}\end{array}$ & $\begin{array}{c}\text { Inhalationa } \\
1 \mathrm{MgSO}_{4} \\
\text { then } \\
\text { Sildenafil } \\
(\mathrm{N}=12) \\
\text { B } \\
\end{array}$ & $\begin{array}{c}\text { Inhalational } \\
\text { MgSO }_{4} \\
\text { with } \\
\text { Sildenafil } \\
(\mathrm{N}=12) \\
\mathrm{C} \\
\end{array}$ & & & & \\
\hline $\begin{array}{l}\text { Duration of treatment } \\
\text { (days) } \\
\text { Mean } \pm \text { S.D. } \\
\text { Median (Range) }\end{array}$ & $\begin{array}{c}4.15 \pm 1.19 \\
4(2-7)\end{array}$ & $\begin{array}{c}5.17 \pm 1.12 \\
5(4-7)\end{array}$ & $\begin{array}{l}6.92 \pm 1.68 \\
6.5(5-10)\end{array}$ & $\begin{array}{c}<0.001 \\
*\end{array}$ & $\begin{array}{c}\mathbf{0 . 0 2 2} \\
* \\
\end{array}$ & $\begin{array}{c}<0.001 \\
*\end{array}$ & $\begin{array}{c}\mathbf{0 . 0 0 8} \\
*\end{array}$ \\
\hline $\begin{array}{l}\mathrm{SpO}_{2}(\%) \\
\text { Mean } \pm \text { S.D. } \\
\text { Median (Range) } \\
\end{array}$ & $\begin{array}{l}94.58 \pm 2.79 \\
95(89-99) \\
\end{array}$ & $\begin{array}{r}92.5 \pm 1.31 \\
93(90-94)\end{array}$ & $\begin{array}{c}91.75 \pm 2.3 \\
90.5(90-96) \\
\end{array}$ & $0.005 *$ & $\begin{array}{l}\mathbf{0 . 0 1 7} \\
* \\
\end{array}$ & $0.005^{*}$ & 0.341 \\
\hline $\begin{array}{l}\text { Pulmonary pressure } \\
(\mathbf{m m H g}) \\
\text { Mean } \pm \text { S.D. } \\
\text { Median (Range) }\end{array}$ & $\begin{array}{c}30 \pm 0 \\
30(30-30)\end{array}$ & $\begin{array}{c}30 \pm 0 \\
30(30-30)\end{array}$ & $\begin{array}{c}30 \pm 0 \\
30(30-30)\end{array}$ & 1 & 1 & 1 & 1 \\
\hline $\begin{array}{l}\text { ABG } \\
\text { Normal } \\
\text { Abnormal } \\
\end{array}$ & $\begin{array}{c}26(100 \%) \\
0(0.0 \%)\end{array}$ & $\begin{array}{c}12(100 \%) \\
0(0.0 \%)\end{array}$ & $\begin{array}{c}12(100 \%) \\
0(0.0 \%)\end{array}$ & NA & NA & NA & NA \\
\hline $\begin{array}{l}\text { Outcome } \\
\text { Dead } \\
\text { Live } \\
\end{array}$ & $\begin{array}{c}2(7.7 \%) \\
24(92.3 \%) \\
\end{array}$ & $\begin{array}{c}2(16.7 \%) \\
10(83.3 \%)\end{array}$ & $\begin{array}{c}2(16.7 \%) \\
10(83.3 \%) \\
\end{array}$ & 0.621 & 0.577 & 0.577 & 1 \\
\hline $\begin{array}{l}\text { Other morbidities } \\
\text { NEC } \\
\text { Sepsis } \\
\text { Sepsis \& NEC } \\
\text { No }\end{array}$ & $\begin{array}{c}3(11.5 \%) \\
2(7.7 \%) \\
3(11.5 \%) \\
18(69.3 \%)\end{array}$ & \begin{tabular}{l||}
$0(0.0 \%)$ \\
$2(16.7 \%)$ \\
$2(16.7 \%)$ \\
$8(66.7 \%)$
\end{tabular} & $\begin{array}{l}2(16.7 \%) \\
4(33.3 \%) \\
2(16.7 \%) \\
4(33.3 \%)\end{array}$ & 0.295 & 0.535 & 0.134 & 0.261 \\
\hline $\begin{array}{l}\text { Supportive treatment } \\
\text { CPAP } \\
\text { Oxygen }\end{array}$ & $\begin{array}{l}12(46.2 \%) \\
14(53.8 \%)\end{array}$ & $\begin{array}{l}6(50 \%) \\
6(50 \%) \\
\end{array}$ & $\begin{array}{l}9(75 \%) \\
3(25 \%)\end{array}$ & 0.24 & 0.825 & 0.096 & 0.4 \\
\hline
\end{tabular}

Table(6): Comparison between the three studied groups regarding duration \& outcome of treatment and supportive treatment.

$P$-value compared the three groups.

p1 a comparison between group $A$ and group $B$.

$P 2$ a comparison between group $A$ and group $C$

$P 3$ a comparison between group $B$ and group $C$ 
SOHAG MEDICAL JOURNAL Inhalational versus intravenous magnesium sulfate in management

*Statistically significant

NA (not applicable)

When we talk about the duration of treatment, it appears that there's a statistically significant difference between the studied groups. In group A it was (2_7) days, while in group B it was (4_7) days and in group $\mathrm{C}$ it was (5_10) days.

$\mathrm{SpO} 2$ after the treatment also differs significantly in the studied groups, in group A mean oxygen saturation was 94.58 while in group B was 92.5 and in the group, $\mathrm{C}$ was 91.75

PAP in the three studied groups after treatment was equal to $30 \mathrm{mmHg}$ as it was the cut-off point for termination of treatment, and therefore there was no statistically significant difference between them.

ABG also was normal in $100 \%$ of cases after treatment.
As regards the mortality rate in the studied groups, it was $7.7 \%$ of group A and $16.7 \%$ in both groups B and C.

Other morbidities which included NEC and $\backslash$ or sepsis, occurred in $30.7 \%$ of group A, $33.3 \%$ of group B and $66.7 \%$ of group C.

Patients received supportive treatment in the form of CPAP were $46.2 \%$ of group A, $50 \%$ of group B and $75 \%$ of group $\mathrm{C}$, while who received supportive treatment in the form of Oxygen were $58.3 \%$ of group A, $50 \%$ of group B and 25\% of group C.

There was a statistically insignificant difference between the studied groups as regards $A B G$, mortality rate, other morbidities, and supportive treatment.

\begin{tabular}{|c|c|c|c|}
\hline Group & $\begin{array}{l}\mathrm{SpO}_{2}(\mathrm{RT} \\
\text { hand) } \\
\text { before the } \\
\text { treatment }\end{array}$ & $\begin{array}{l}\text { Post- } \\
\text { treatment } \\
\mathrm{SpO}_{2}(\%)\end{array}$ & P-value \\
\hline \multicolumn{4}{|l|}{ I.V $\mathrm{MgSO}_{4}(\mathrm{~A})$} \\
\hline $\begin{array}{l}\text { Mean } \pm \text { S.D. } \\
\text { Median (Range) }\end{array}$ & $\begin{array}{l}79.15 \pm 8.18 \\
80.5(60-90)\end{array}$ & $\begin{array}{l}94.58 \pm 2.79 \\
95(89-99)\end{array}$ & $<0.001 *$ \\
\hline \multicolumn{4}{|c|}{$\begin{array}{l}\text { Inhalational } \mathrm{MgSO}_{4} \\
\text { then Sildenafil (B) }\end{array}$} \\
\hline $\begin{array}{l}\text { Mean } \pm \text { S.D. } \\
\text { Median (Range) }\end{array}$ & $\begin{array}{l}83.42 \pm 3.15 \\
83(79-90)\end{array}$ & $\begin{array}{l}92.5 \pm 1.31 \\
93(90-94)\end{array}$ & $0.002 *$ \\
\hline \multicolumn{4}{|c|}{$\begin{array}{l}\text { Inhalational } \mathrm{MgSO}_{4} \\
\text { with Sildenafil (C) }\end{array}$} \\
\hline $\begin{array}{l}\text { Mean } \pm \text { S.D. } \\
\text { Median (Range) }\end{array}$ & $\begin{array}{l}79.92 \pm 5.19 \\
80.5(70-86)\end{array}$ & $\begin{array}{l}91.75 \pm 2.3 \\
90.5(90-96)\end{array}$ & $0.002 *$ \\
\hline
\end{tabular}

Table (7): Comparison of SpO2 (RT hand) before the treatment and post-treatment $\mathrm{SpO} 2(\%)$ in the study groups

P-value was calculated by Wilcoxon Signed Ranks test *Statistically significant

When we compared $\mathrm{SpO} 2$ in the right hand before and after treatment there was a statistically significant difference in all studied groups; in group A the mean $\mathrm{SpO} 2$ before treatment was 79.15 while after treatment was 94.58. In group $\mathrm{B}$, mean $\mathrm{SpO} 2$ before treatment was 83.42 while after treatment was 92.5. In group $\mathrm{C}$, mean $\mathrm{SpO} 2$ before treatment was 79.92 while after treatment was 91 
SOHAG MEDICAL JOURNAL Inhalational versus intravenous magnesium sulfate in management

\begin{tabular}{|c|c|c|c|}
\hline Group & $\begin{array}{l}\text { Pulmonary } \\
\text { pressure before } \\
\text { treatment } \\
\end{array}$ & $\begin{array}{l}\text { Pulmonary } \\
\text { pressure post- } \\
\text { treatment } \\
\end{array}$ & P-value \\
\hline \multicolumn{4}{|l|}{$\mathrm{I}^{\mathrm{V} \mathrm{MgSO}} \mathrm{Mg}_{4}(\mathrm{~A})$} \\
\hline $\begin{array}{l}\text { Mean } \pm \text { S.D. } \\
\text { Median (Range) }\end{array}$ & $\begin{array}{l}69.81 \pm 17.51 \\
70(50-110) \\
\end{array}$ & $\begin{array}{l}30 \pm 0 \\
30(30-30) \\
\end{array}$ & $<0.001 *$ \\
\hline \multicolumn{4}{|c|}{$\begin{array}{l}\text { Inhalational } \mathrm{MgSO}_{4} \\
\text { then Sildenafil (B) }\end{array}$} \\
\hline $\begin{array}{l}\text { Mean } \pm \text { S.D. } \\
\text { Median (Range) }\end{array}$ & $\begin{array}{l}57.08 \pm 4.98 \\
60(50-65) \\
\end{array}$ & $\begin{array}{l}30 \pm 0 \\
\mathbf{3 0}(\mathbf{3 0}-\mathbf{3 0}) \\
\end{array}$ & 0.002* \\
\hline \multicolumn{4}{|c|}{$\begin{array}{l}\text { Inhalational } \mathrm{MgSO}_{4} \\
\text { with Sildenafil }(\mathrm{C}) \\
\end{array}$} \\
\hline $\begin{array}{l}\text { Mean } \pm \text { S.D. } \\
\text { Median (Range) }\end{array}$ & $\begin{array}{l}78.17 \pm 8.04 \\
80(65-90)\end{array}$ & $\begin{array}{l}30 \pm 0 \\
30(30-30)\end{array}$ & 0.002* \\
\hline
\end{tabular}

Table (8): Comparison of pulmonary pressure before and after treatment in the study groups.

P-value was calculated by Wilcoxon Signed Ranks test *Statistically significant

This table shows the difference in PAP before and after treatment with an obvious statistically significant difference between them. In the group, A mean pulmonary pressure before treatment was $69.81 \mathrm{mmHg}$ while after treatment was $30 \mathrm{mmHg}$. In the group, B mean pulmonary pressure before treatment was $57.08 \mathrm{mmHg}$ while after treatment was $30 \mathrm{mmHg}$. In the group, $\mathrm{C}$ mean pulmonary pressure before treatment was $78.17 \mathrm{mmHg}$ while after treatment was $30 \mathrm{mmHg}$.

\section{Discussion}

Davidson et al. [6] in their study showed that early I-NO as the sole adjunct to conventional management produced acute and sustained improvement in oxygenation for 24 hours without short-term side effects [5 and $20 \mathrm{ppm}$ doses], and the suggestion that ECMO use may be reduced. In two large randomized studies, so reduced the need for ECMO support by about $40 \%$. However, it did not reduce mortality, the duration of hospitalization, or the risk of neurodevelopmental impairment [7]. Currently, the initial recommended dose of iNO is $20 \mathrm{ppm}$. Higher doses have the same efficacy and are associated with an increased risk of methemoglobinemia and the formation of nitrogen dioxide [8].

However, unfortunately, so is not available in our country and we have to use other available treatment modalities such as $\mathrm{MgSO} 4$ and sildenafil for treating patients with PPHN.

In this study, we tried to evaluate the response to nebulized magnesium sulfate as a new modality of treatment in PPHN. Magnesium sulfate enhances vasodilatation by promoting the entry of calcium ions into the smooth muscle cells. MgSO4 was listed as a safe and effective pulmonary vasodilator according to Chandran et al. [9].

$\mathrm{MgSO} 4$ can be used as a first-line pulmonary vasodilator because it has a low cost and high efficacy. IV MgSO4 can be used and monitored easily during treatment. However, systemic hypertension occurs more with IV $\mathrm{MgSO}_{4}$ [10].

In patients with acute asthma, isotonic magnesium sulfate, as a vehicle for nebulized salbutamol, increased the peak flow response to treatment in comparison with salbutamol plus normal saline according to Nannini et al. [11].

However the use of nebulized MgSO4 as a pulmonary vasodilator hasn't been tried yet worldwide, and we concluded to this result after great effort in search in many books, researches, and articles that were published internationally. 
So we conducted this study based on two facts, the first was the approved efficacy of parenteral $\mathrm{MgSO} 4$ as a bronchodilator and vasodilator also as mentioned above, and the second was the documented use of nebulized $\mathrm{MgSO} 4$ as a bronchodilator in management of mild to severe asthma and its acute exacerbations.

Also, nebulized $\mathrm{MgSO} 4$ would have the advantage of avoiding systemic hypotension that occurs with intravenous $\mathrm{MgSO} 4$.

The three studied groups were compared to each other as regard age, sex, anthropometric measures, maternal history, and neonatal examination before and after treatment, laboratory investigations, chest X-ray, echo before and after treatment, side effects of treatment, duration and outcome of treatment.

As regard age and sex, P-value was 0.034 and 0.001 respectively between the three studied groups which are statistically significant. There was also a statistically significant difference between the three studied groups concerning the degree of respiratory distress [P value 0.01], the pulmonary artery pressure before treatment $[\mathrm{P}$ value $<0.001]$, side effects of treatment [P value 0.012$]$, duration of treatment $[\mathrm{P}$ value $<0.001]$ and the $\mathrm{SpO} 2$ after treatment [P value 0.005].

In our study the number of neonates in group A was 26 with male: female ratio $1: 1$ and the number in group $B$ was 12 with male: female ratio 1:5 and 12 patients in group $\mathrm{C}$ and male: female ratio was 11:1 while the result of Uslu et al. [12] was somewhat different, it included 34 patients in intravenous $\mathrm{MgSO} 4$ group with male: female ratio $1.44: 1$ and 31 patients in oral sildenafil group with male: female ratio $1.1: 1$ but different to the result of Dhariwal et al. [13] whose male: female ratio was 5:1
The mean age in group A was 2.5 days while in group B was 4.3 days and in the group, $\mathrm{C}$ was 1.8 days which was very close to the result of Uslu $\boldsymbol{e t}$ al.[12] where the median age was 2 days in each group but quite far from the results of Steinhorn et al. [14] where the mean age was 34 hours.

As regard maternal DM in our study $8 \%$ of neonates were infants of diabetic mothers but it was $5 \%$ in the study of Razzaq et al. [15] maternal hypertension andlor PET was positive in $6 \%$ of cases while it was positive in only $3.8 \%$ of cases in the study of Razzaq et al. [15].

Also, the study of Delaney et al. [16] showed that maternal DM and PET are among the factors associated with an increased risk for PPHN. Maternal PROM didn't occur at all in our study just like the results of HernándezDíaz [17].

In our study, the mean neonatal weight was $2.9 \mathrm{~kg}$ in both group A \& $\mathrm{C}$ and 2.7 in group B that's near the neonatal weight in the study of Uslu $\boldsymbol{e t}$ al. [12] that was $3.2 \mathrm{~kg}$ and the study of Nakwan et al. [18] that was $3.4 \mathrm{~kg}$ The mean height in our study was 49.7 $\mathrm{cm}$ and the mean head circumference was $34.6 \mathrm{~cm}$ in all groups that were very close to the mean height and head circumference in the study of Uslu $\boldsymbol{e t}$ al. [12] which were $49.9 \mathrm{~cm}$ and $35 \mathrm{~cm}$ respectively and $49.8 \mathrm{~cm}$ and $34.8 \mathrm{~cm}$ in the study of Nakwan N. et al. [18]. Respiratory distress was found in $100 \%$ of cases in our study that's the same as in Uslu et al. study. [12] and near the result of Tissot et al. [19] that was $86 \%$

Down syndrome was found in only $2 \%$ of neonates in our study and also hydrocephalus that's quite near to the results of Cua et al. [20] that showed that $1.2 \%$ of cases had down syndrome but none had hydrocephalus.

In our study, the mean $\mathrm{SpO} 2$ was $79.15 \%$ in group A and $83.4 \%$ in group 
$\mathrm{B}$ and $79.9 \%$ in group $\mathrm{C}$ which was higher than the results of Uslu et al. [12] in which $\mathrm{SpO} 2$ was $64.3 \%$ in the $\mathrm{MgSO} 4$ group and $66 \%$ in the sildenafil group.

Heart sounds were normal in $80 \%$ of cases in this study while only $20 \%$ had single loud S2 and also 38\% of cases had the murmur of tricuspid regurgitation. This result is different from the result of Bakheet et al. [21] in which $88 \%$ of cases had single loud S2.

As regard laboratory investigations in our study $90 \%$ of cases had normal CBC, 92\% had normal blood glucose levels and $90 \%$ had normal serum electrolytes which is very close to the results of Hageman et al. [22] in which CBC was normal in $93 \%$ of cases, and normal blood glucose and serum electrolytes in the vast majority of cases and Razzaq et al. [15] study that had $96.9 \%$ of cases with normal CBC.

The ABG in our study showed only $6 \%$ of cases had compensated metabolic acidosis, $40 \%$ had uncompensated metabolic acidosis and $100 \%$ of cases had profound hypoxemia which completely agrees with results of Uslu et al. [12] and Razzaq et al. [15] that showed that $100 \%$ of cases had profound hypoxemia.

Chest X-ray findings in our study were normal in $64 \%$ of cases that is agree with the study of Sharma et al. [10] that show that lung fields are clear or minimally opacified on X-ray but in Bakheet et al. study [21] show 38\% had cardiomegaly.

The PAP in our study had a mean of $69.8 \mathrm{mmHg}$ in group $\mathrm{A}, 57.08 \mathrm{mmHg}$ in group B and $78.17 \mathrm{mmHg}$ in group $\mathrm{C}$ which is much higher than that of Uslu et al. [12] that was $45.6 \mathrm{mmHg}$ in the $\mathrm{MgSO} 4$ group and $46.2 \mathrm{mmHg}$ in the sildenafil group. Also, the median of PAP in our study which was 70
$\mathrm{mmHg}$ in all groups is much higher than the median of PAP in the study of Milman et al. [23] that was $36 \mathrm{mmHg}$. Time taken to achieve adequate response in our study was 2 - 7 days in group A, 4 - 7 days in group B and 5 10 days in group $C$ and this result is somewhat near to the result of Uslu, et al. [12] that was 1 - 12 days in $\mathrm{MgSO} 4$ group and 1 - 11 days in the sildenafil group. The mean duration of treatment was 3.1 days in the study of Tolsa $\boldsymbol{e t}$ al. [24] which is nearly the same as our result which was 3.4 days.

The cut-off point of PAP in this study was $30 \mathrm{mmHg}$ which was achieved in all patients but in different durations and supportive measures either CPAP or inhaled oxygen, while the PAP cut off point in Uslu et al. [12] study was $20 \mathrm{mmHg}$.

Mortality rate in this study was $7.7 \%$ in group A and $16.7 \%$ in both group $\mathrm{B} \& \mathrm{C}$ while it was $5.9 \%$ in $\mathrm{MgSO} 4$ group and $3.2 \%$ in the sildenafil group in Uslu et al. [12] study, and was 11\% in the study of Michele et al. [25] which is probably due to the larger number of cases in their studies.

Associated morbidities in our study included sepsis which occurred in 30\% of cases in all groups while sepsis occurred in $29.1 \%$ of cases in the study of Razzaq et al. [15] that's approximately the same.

$54 \%$ of our cases used CPAP as a supportive treatment and $46 \%$ used Oxygen only while in the study of Razzaq et al. [15] 64.5\% of cases used CPAP which is very close to our results, $15.1 \%$ used Oxygen and the rest $20.4 \%$ used intermittent mandatory ventilation which hasn't been used in our study.

Oral sildenafil was used in group $\mathrm{B} \& \mathrm{C}$ as an associated treatment because of its proved efficacy as it is a phosphodiesterase type 5 inhibitor which reduces the pulmonary vascular resistance selectively as proved by 
Hernando Baquero et al. [26].in the present study $\mathrm{s}$ was added to patients who didn't respond to inhalational $\mathrm{MgSO} 4$ for 24 hours and their general condition became worse and then we decided to use it from the start as an adjuvant treatment in patients with higher pulmonary pressure to save their lives.

So this study was the first trial to evaluate the response to nebulized magnesium sulfate as a new modality of treatment in PPHN. We just opened a window for further researches to be held on this topic.

\section{Limitations of this study included:}

- The small number of patients.

- Calculation of the dose of nebulized MgSO4 was based on our trials but no documented dose was used as this route of administration hasn't been tried yet before, so dose adjustment might have some fallacies.

- Nebulizers available in our study are of the pneumatic type which is much less efficient than ultrasonic nebulizers.

\section{Recommendation:}

So, based on this study we don't recommend the use of nebulized $\mathrm{MgSO} 4$ alone as a treatment of PPHN in neonates (as no improvement occurred in patients used it alone) and use other treatment modalities available in our country until other researches or studies can prove the reverse of our results.

\section{References:}

1. Abman SH, Chatfield BA, Hall SL, McMurtry IF. Role of an endotheliumderived relaxing factor during the transition of pulmonary circulation at birth. American Journal of PhysiologyHeart and Circulatory Physiology. 1990 Dec 1;259(6): H1921-7.

2. Konduri GG, Kim UO. Advances in the diagnosis and management of persistent pulmonary hypertension of the newborn. Pediatric Clinics. 2009 Jun 1;56(3):579-600.

3. Finer NN, Barrington KJ. Nitric oxide for respiratory failure in infants born at or near term. Cochrane Database Syst Rev. 2006 Oct 18;4(4): CD000399.

4. Chandran S, Haqueb ME, Wickramasinghec HT, Wint Z. Use of magnesium sulfate in severe persistent pulmonary hypertension of the newborn. Journal of tropical pediatrics. 2004 Aug 1;50(4):219-23.

5. Tolsa JF, Cotting J, Sekarski N, Payot M, Micheli JL, Calame A. Magnesium sulfate as an alternative and safe treatment for severe persistent pulmonary hypertension of the newborn. Archives of Disease in Childhood-Fetal and Neonatal Edition. 1995 May 1;72(3): F184-7.

6. Davidson D, Barefield ES, Kattwinkel J, Dudell G, Damask M, Straube R, Rhines J, Chang CT, I-NO/PPHN Study Group. Inhaled nitric oxide for the early treatment of persistent pulmonary hypertension of the term newborn: a randomized, doublemasked, placebo-controlled, doseresponse, multicenter study. Pediatrics. 1998 Mar 1;101(3):325-34.

7. Fuloria M, Aschner JL. Persistent pulmonary hypertension of the newborn. InSeminars in Fetal and Neonatal Medicine 2017 Aug 1 (Vol. 22, No. 4, pp. 220-226). WB Saunders.

8. Lemons JA, Blackmon LR, Kanto WP, Macdonald HM, Miller CA, Rosenfeld W, Shoemaker CT, Stewart JE, Speer ME, Greene MF, Johnson P. Use of inhaled nitric oxide. Pediatrics. 2000 Aug 1;106(2):344-5.

9. Chandran S, Haqueb ME, Wickramasinghec HT, Wint Z. Use of magnesium sulfate in severe persistent pulmonary hypertension of the newborn. Journal of tropical pediatrics. 2004 Aug 1;50(4):219-23.

10.Sharma M, Mohan KR, Narayan S, Chauhan L. Persistent pulmonary hypertension of the newborn: a review. Medical Journal Armed Forces India. 2011 Oct 1;67(4):348-53.

11.Nannini Jr LJ, Pendino JC, Corna RA, Mannarino S, Quispe R. Magnesium 
sulfate as a vehicle for nebulized salbutamol in acute asthma. The American journal of medicine. 2000 Feb 15;108(3):193-7.

12.Uslu S, Kumtepe S, Bulbul A, Comert S, Bolat F, Nuhoglu A. A comparison of magnesium sulfate and sildenafil in the treatment of the newborns with persistent pulmonary hypertension: a randomized controlled trial. Journal of tropical pediatrics. 2010 Oct 4;57(4):245-50.

13.Dhariwal AK, Bavdekar SB. Sildenafil in pediatric pulmonary arterial hypertension. Journal of postgraduate medicine. $2015 \mathrm{Jul} ; 61(3): 181$.

14.Steinhorn RH, Kinsella JP, Pierce C, Butrous G, Dilleen M, Oakes M, Wessel DL. Intravenous sildenafil in the treatment of neonates with persistent pulmonary hypertension. The Journal of pediatrics. 2009 Dec 1;155(6):841-7.

15.Razzaq A, Quddusi AI, Nizami N. Risk factors and mortality among newborns with persistent pulmonary hypertension. Pakistan journal of medical sciences. 2013 Sep;29(5):1099.

16.Delaney C, Cornfield DN. Risk factors for persistent pulmonary hypertension of the newborn. Pulmonary circulation. 2012 Jan;2(1):15-20.

17.Hernández-Díaz $S$, Van Marter LJ, Werler MM, Louik C, Mitchell AA. Risk factors for persistent pulmonary hypertension of the newborn. Pediatrics. 2007 Aug 1;120(2):e272-82.

18. Nakwan N, Nakwan N, Wannaro J. Persistent pulmonary hypertension of the newborn successfully treated with beraprost sodium: a retrospective chart review. Neonatology. 2011;99(1):32-7.

19. Tissot C, Ivy DD, Beghetti M. Medical therapy for pediatric pulmonary arterial hypertension. The Journal of pediatrics. 2010 Oct 1;157(4):528-32.

20.Cua CL, Blankenship A, North AL, Hayes J, Nelin LD. Increased incidence of idiopathic persistent pulmonary hypertension in Down syndrome neonates. Pediatric cardiology. 2007 Aug 1;28(4):250-4.

21. Bakheet MA, Metwalley KA, AbdelRaheem AS. Evaluation of persistent pulmonary hypertension of the newborn (PPHN) in Upper Egypt. Egyptian Pediatric Association Gazette. 2013;3(61):96-9.

22.Hageman JR, Adams MA, Gardner TH. Persistent pulmonary hypertension of the newborn: trends in incidence, diagnosis, and management. American Journal of Diseases of Children. 1984 Jun 1;138(6):592-5.

23. Milman N, Svendsen CB, Iversen M, Videbæk R, Carlsen J. Sarcoidosisassociated pulmonary hypertension: acute vaso-responsiveness to inhaled nitric oxide and the relation to the longterm effect of sildenafil. The clinical respiratory journal. 2009 Oct;3(4):20713.

24.Tolsa JF, Cotting J, Sekarski N, Payot M, Micheli JL, Calame A. Magnesium sulfate as an alternative and safe treatment for severe persistent pulmonary hypertension of the newborn. Archives of Disease in Childhood-Fetal and Neonatal Edition. 1995 May 1;72(3): F184-7.

25.Walsh-Sukys MC, Tyson JE, Wright LL, Bauer CR, Korones SB, Stevenson DK, Verter J, Stoll BJ, Lemons JA, Papile LA, Shankaran S.

26.Persistent pulmonary hypertension of the newborn in the era before nitric oxide: practice variation and outcomes. Pediatrics. 2000 Jan 1;105(1):14-20.

27.Baquero H, Soliz A, Neira F, Venegas ME, Sola A. Oral sildenafil in infants with persistent pulmonary hypertension of the newborn: a pilot randomized blinded study. Pediatrics. 2006 Apr 1;117(4):1077-83. 